
ISSN 1813-548X

\title{
Morphodynamisme des environnements à Beach-rocks du plateau continental de Côte d'Ivoire (golfe de Guinée)
}

Sylvain MONDE*, Colette A. AKOBE, Bruno Z. DIGBEHI et Kouamé AKA

Université de Cocody, UFR Sciences de la Terre et des Ressources Minières, Laboratoire de Géologie Marine et de Sédimentologie - GEOMARSE

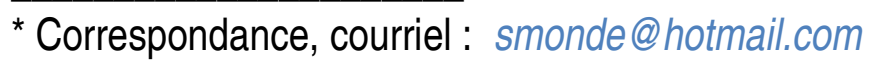

\section{Résumé}

Les beach-rocks sont constituées de grains de quartz, avec une faible proportion de feldspath et de mica. II s'agit de grès quartzeux à ciment ferrugineux, parfois carbonatés. Ils sont constitués de matériaux terrigènes charriés par les cours d'eau. Ces grès ont subi un long transport et une cimentation par précipitation de l'oxyde de fer. L'absence de fraction argileuse, la faible proportion de feldspaths et de micas, et l'abondance de quartz à grains arrondis indiquent qu'ils sont matures. En effet, la présence de quartzs polycristallins indiquant une recristallisation et une substitution du ciment, montre que ces grès ont subi une diagenèse avancée. La fraction sableuse adjacente aux barres de grès du plateau continental montre des sables d'origine fluviatile, mal triés à classement médiocre présentent une asymétrie vers les plus petites tailles. Ces grès se déposeraient dans un environnement de type ligne de rivage, sous l'influence de courants locaux bimodaux dus aux variations du niveau de la mer. En raison de leur résistance mécanique et de leur épaisseur, les beach-rocks constituent un obstacle au transport des sédiments.

Mots-clés : Beach-rocks, sédimentologie, paléoenvironnement, plateau continental, Côte d'lvoire 


\section{Abstract \\ Morphodynamism of the beach-rocks environments in the continental shelf of Côte d'Ivoire (gulf of Guinea)}

The beach-rocks are constituted of quartz grain with a low proportion of feldspar and mica. Those concern sandstones with ferruginous cement often carbonated. They are constituted of terrigene materials carried by water streams. These sandstones underwent a long transport and a cementation by iron oxide precipitation. Absence of clayey fractions, the low proportion of feldspar and micas, and the abundance of quartz with round grains indicate the presence of mature beach-rocks. The polycrystalline quartz indicates a recrystallization and a cement substitution shows that sandstones were subjected to an advanced digenesis. The sandy fraction skewed to the beach-rocks of the continental shelf shows that, sands are from fluvial origin, poorly sorted with a low scaling and present an asymmetry toward the smallest sizes. These sandstones were established in a curve line environment under the influence of local bimodal streams due to variation in sea level. Due to their mechanic resistance and their thickness, beach-rocks constitute an important obstacle for sediments transportation.

Keywords : Beach-rock, sedimentlogy, palaeoenvironment, continental shelf, Côte d'lvoire

\section{Introduction}

Les beach-rocks constituent l'un des traits morphologiques caractéristiques des plateaux continentaux du Golfe de Guinée. Ces formations s'étendent parallèlement à la ligne de côte et rompent la monotonie de la surface relativement plane des plates-formes continentales. II s'agit de cordons littoraux fossiles, constitués de sables à ciment calcaire et de débris coquilliers. Les sondages sismiques montrent que les beach-rocks sont présents sur l'ensemble du littoral du Bénin à des profondeurs variables [1]. Sur les côtes du Togo, les barres 
de grès se présentent en dalles superposées qui affleurent en marée basse. Leur épaisseur varie entre 0,5 et $1 \mathrm{~m}$ sur une largeur d'environ 25 à $50 \mathrm{~m}$ [2]. En Côte d'Ivoire, les beach-rocks constituent des écueils à la navigation et rendent difficile la pêche en détruisant les chaluts. Ils se retrouvent sur l'ensemble du plateau continental ivoirien à des profondeurs de 45 à $110 \mathrm{~m}$. Ces bancs rocheux atteignent une hauteur de 1,5 à $20 \mathrm{~m}$ avec une largeur de $200 \mathrm{~m}$ à $1 \mathrm{~km}[3,4]$. En outre, ils sont associés aux profiles bathymétriques concaves du plateau continental. Ces ensembles morphologiques s'observent dans les régions sableuses, entre les zones d'apports sédimentaires actuels et ils présentent le plus souvent des ruptures de pentes correspondant à l'emplacement des beach-rocks qui étaient masqués par la couverture vaseuse dans les types bathymétriques convexes et intermédiaire [5 - 7]. Les profils convexes, aux larges des embouchures des grands fleuves, présentent une pente légèrement accentuée au départ de la côte, puis elle passe ensuite par un palier et enfin s'accentue à nouveau jusqu'au rebord du plateau. Entre les ensembles morphobathymétriques convexes et concaves, se rencontrent des ensembles intermédiaires, localisés sur les bordures de vasières ou sur les vasières à faibles apports sédimentaires actuels.

De récentes campagnes d'échantillonnages de sédiments, dont Chalci 94 (du Centre de Recherches Océanologiques d'Abidjan -1994) et Sédicot 98 (de l'Université de Cocody -1998), ont été réalisées sur ces trois ensembles bathymétriques. Des résultats issus de ces études, la présente note fait une analyse pétro-sédimentaire des faciès superficiels meubles et des beach-rocks du plateau continental ivoirien. En outre, elle propose une interprétation de leur paléoenvironnement dans le Golfe de Guinée.

\section{Matériel et méthodes}

L'échantillonnage des sédiments dans la plate-forme de Côte d'lvoire (Figure 1), s'est déroulé selon 20 radiales, d'Assinie $\left(3^{\circ} 20^{\prime} \mathrm{W}\right)$ à Tabou $\left(7^{\circ} 10^{\prime} \mathrm{W}\right)$. Toutefois seules les radiales de Dabégo $\left(6^{\circ} 00^{\prime} \mathrm{W}\right)$, de Fresco 
$\left(5^{\circ} 25^{\prime} \mathrm{W}\right)$ et d'Alékedon $\left(5^{\circ} 15^{\prime} \mathrm{W}\right)$ présentent un intérêt pour l'étude des beach-rocks car elles correspondent aux zones morphobathymétriques concaves. Les prélèvements des sédiments ont été effectués avec une benne pour les sédiments meubles et une drague à roches pour les beach-rocks.

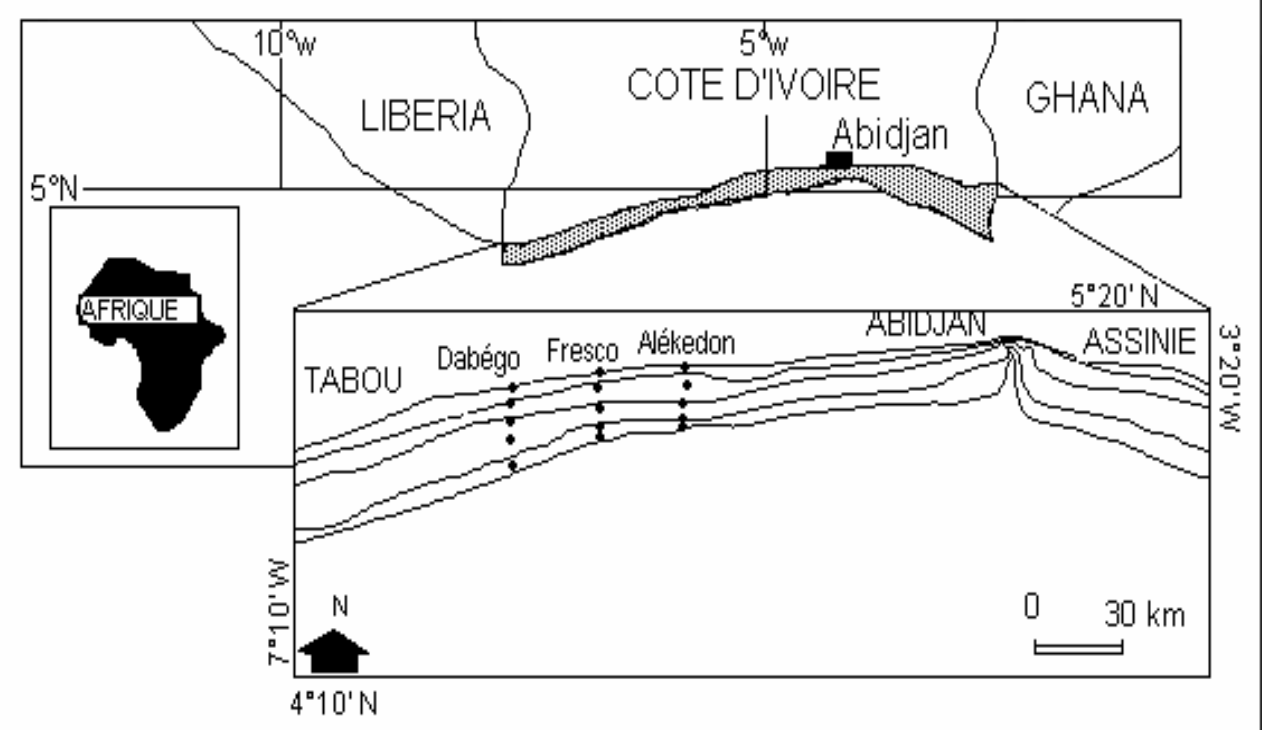

Figure 1 : Environnements à beach-rocks de Côte d'Ivoire. Zones à morphobathymétrie concave du plateau continental à Alékedon, Fresco et Dabégo.

Le traitement des échantillons s'est effectué en deux phases:

(i) la description de la coloration des sédiments se base sur l'échelle standard de coloration du Munsell color system of Geological Society of America [8],

(ii) l'analyse granulométrique porte sur la fraction meuble. Celle-ci après conditionnement (lavage et séparation de la fraction sableuse, attaques chimiques au $\mathrm{HCl}$ et $\mathrm{H}_{2} \mathrm{O}_{2}$, séchage à l'étuve) est tamisée dans une colonne de tamis (12) vibrants (Afnor) de mailles comprises entre $63 \mu \mathrm{m}$ et $1,25 \mathrm{~mm}$. 
La nature lithologique des sédiments meubles adjacents aux beach-rocks a été déterminée grâce au diagramme de Chamley [9]. Ce diagramme ternaire présente une nomenclature qui est fonction des proportions de sable, de silt et d'argile contenues dans le sédiment. En outre, deux autres classifications ont permis de caractériser les barres de grès:

(i) la classification de Cailleux [10] intègre dans la terminologie des différents types de grès la nature du ciment et des minéraux exceptionnels,

(ii) la classification de Dott [11] modifiée par Pettijohn et al. [12] utilise un diagramme ternaire dont les sommets correspondent aux proportions en quartz, en feldspath et en lithite du sédiment.

L'étude pétrographique des barres de grès est faite à l'échelle macro et microscopique. Une telle approche permettra, la connaissance de la nature lithologique et de la classe pétrographique des beach-rocks. Ces approches précisent l'origine, le mode de dépôt, l'environnement de dépôt et la maturité des barres de grès en vue d'une interprétation paléoenvironnementale.

\section{Résultats et discussion}

\section{3-1. Environnement de dépôt des sédiments adjacents aux beach- rocks}

L'analyse des sédiments superficiels du plateau continental de Côte d'Ivoire présente trois faciès lithologiques (Tableau 1) qui s'observent sur les ensembles morphobathymétriques concaves :

(i) les sables ont une granulométrie moyenne à grossière. Ils ont une couleur brun-jaunâtre à degré variable,

(ii) les vases, de couleur vert-olive-grisâtre et de compacité variable, se rencontrent sur tout le plateau continental,

(iii) les sédiments «mixtes» renferment du sable et de la vase en proportions variables. Les sables vaseux contiennent 50 à $75 \%$ d'éléments de diamètre supérieur à $0,05 \mathrm{~mm}$ avec des fractions granulométriques variables (sables vaseux grossiers, sables vaseux 
moyens et sables vaseux fins). Les autres fractions mixtes sont représentées par les vases sableuses. L'évolution granulométrique des sédiments mixtes indique la prédominance des formes fines (35\%) et très fines (34\%) au regard des grains moyens avec $22 \%$ [13].

La radiale Dabégo $\left(6^{\circ} 00^{\prime} \mathrm{W}\right)$ présente $100 \%$ de grains fins jusqu'à la première moitié du plateau continental intermédiaire, puis entre -65 et -85 $\mathrm{m}$, les grains grossiers sont de $25 \%$ et les grains fins sont de $75 \%$ (Figure 2a). Dans la partie externe du plateau continental, de -80 à -100 $\mathrm{m}$, les sédiments sont d'abord à grains moyens (25\%) et à grains fins $(75 \%)$. Au-delà de $-100 \mathrm{~m}$, les sédiments à grains moyens prédominent (75\%).

A Alékedon $\left(5^{\circ} 15^{\prime} \mathrm{W}\right)$, la distribution granulométrique montre que $75 \%$ des sédiments sont à grains moyens et $25 \%$ sont à grains grossiers sur les plates-formes interne et intermédiaire (Figure $2 b$ ). Puis, la proportion en grains moyens (25\%) baisse et celle des sédiments à grains fins (75\%) s'accroît dans la plate-forme externe.

Dans la région de Fresco $\left(5^{\circ} 25^{\prime} \mathrm{W}\right)$, l'abondance des sédiments à grains grossiers et moyens est inversement proportionnelle à celle des sédiments fins et très fins (Figure $2 c$ ). En effet, l'évolution des grains fins et très fins est croissante du plateau continental interne au plateau continental externe, à l'inverse des grains grossiers et moyens. Seuls les grains très grossiers sont inexistants $(1 \%)$ sur l'ensemble du plateau continental. 
Tableau 1 : Granulométrie et description des sables des types morphobathymétriques concaves de la plate-forme continentale de Côte d'Ivoire

\begin{tabular}{|c|c|c|c|c|c|c|c|c|c|c|c|c|c|}
\hline Échts. & Position (Lat-Long) & Sonde $(m)$ & Descriptions sédimentologiques & $\operatorname{Stg}^{\star *}$ & $S g$ & $S m$ & Sf & Stf & $M d^{\star \star \star}$ & $M$ & So & Mo & Sk \\
\hline Ch001 & 504,4 ' N - 524'W & 20 & Vase sableuse compacte $/ 5 g 2-1^{*}$ & 1,026 & 5,57 & 28,2 & 41,7 & 20,91 & 0 & 1,29 & 0 & 0,32 & 0 \\
\hline Ch002 & $502,3^{\prime}-523,3^{\prime}$ & 30 & Sable moyen à grossier v aseux /10yr5-4 & 1,03 & 25,6 & 60,4 & 11,4 & 1,2 & 0,17 & 1,64 & 0 & 0,32 & 0 \\
\hline Ch003 & $500,9^{\prime}-524$ & 40 & Vase sableuse /5gy3-2 & 0,15 & 3,7 & 31,4 & 47,8 & 16,53 & 0,69 & 0,62 & 0 & 0,36 & $-0,03$ \\
\hline Ch004 & $459^{\prime}-526,2$ & 50 & Vase sableuse /5gy3-2 & 1,21 & 13,1 & 36,3 & 40 & 9,28 & 0,81 & 0,79 & 0 & 1 & 0,03 \\
\hline Ch005 & $457,7^{\prime}-524,4^{\prime}$ & 65 & Sable moyen vaseux /5gy3 -2 & 4,47 & 17,1 & 39,3 & 32,9 & 5,88 & 0,84 & 0,76 & 0 & 1 & $-0,06$ \\
\hline Ch006 & $455,9^{\prime}-526,4^{\prime}$ & 80 & Vase sableuse compacte / 5gy3-2 & 1,27 & 5,51 & 15,7 & 32 & 43,84 & 1,18 & 1,12 & 0 & 1,32 & 0 \\
\hline Ch007 & $453,8^{\prime}-525,3^{\prime}$ & 100 & Sable vaseux/ 5gy3-2 & 3,13 & 1,79 & 15,6 & 54,7 & 23,53 & 1,29 & 1,32 & 1 & 2,64 & 0,36 \\
\hline Ch008 & $452,8^{\prime}-526,1^{\prime}$ & 110 & Vase sableuse/ 5gy3-2 & 0,07 & 0,22 & 12,4 & 47,5 & 38,39 & 0,54 & 0,51 & 0 & 2,64 & $-0,24$ \\
\hline Ch057 & 4²,2, - 555,3' & 111 & Vase sableuse/ 5g2-1 & 0 & 0,4 & 58,3 & 32,2 & 7,85 & 2,32 & 1,36 & -1 & 2,64 & $-1,1$ \\
\hline Ch058 & 443,3' - 555,7' & 95 & Sable vaseux/ 5gy3-2 & 0,67 & 0,59 & 11,9 & 53,8 & 32,5 & 1,32 & 1,25 & -1 & 1,69 & 0,15 \\
\hline Ch059 & $4{ }^{\circ} 45,5^{\prime}-5{ }^{\circ} 6,6^{\prime}$ & 80 & Sable fin à moyen vaseux / 5gy3-2 & 17,34 & 9,29 & 14,6 & 30,3 & 27,25 & 1,47 & 1,29 & -1 & 1,32 & $-0,15$ \\
\hline Ch060 & $498,3^{\prime}-556,1^{\prime}$ & 65 & Vase sableuse/ 5gy3-2 & 0,939 & 1,11 & 6,99 & 27,8 & 61,2 & 1,22 & 1,25 & 0 & 1 & 0,18 \\
\hline Ch061 & $451,3^{\prime}-555,7^{\prime}$ & 50 & Vase silteuse/ 5gy3-2 & 0 & 0 & 0 & 0 & 0 & - & - & - & - & - \\
\hline Ch062 & $453,2^{\prime}-554$ & 40 & Vase silteuse/ 5gy3-2 & 0 & 0 & 0,69 & 4,27 & 83,33 & 1,69 & 1,74 & -1 & 1,69 & 0,43 \\
\hline Ch063 & $454^{\prime}-556,2$ ' & 30 & Sable fin vaseux/5gy3-2 & 0 & 0,03 & 0,27 & 20,7 & 75,6 & 1,4 & 1,43 & -1 & 1 & 0,36 \\
\hline Ch064 & $457,1^{\prime}-553,7^{\prime}$ & 20 & Sable fin vaseux/ 5gy3-2 & 0 & 0,03 & 0,34 & 10,3 & 87,3 & 1,56 & 1,64 & -1 & 1,69 & 0,56 \\
\hline Ch101 & $455,4^{\prime}-596,8^{\prime}$ & 110 & Vase silteuse avec gale ts argileux/ $5 g 3-2$ & 1,096 & 0,89 & 12,3 & 38,6 & 45,94 & 2,32 & 1,84 & -1 & 2,64 & $-0,3$ \\
\hline Ch102 & $456^{\prime}-597^{\prime}$ & 95 & Sable moyen à grossier vaseu x/ 5gy3-2 & 9,26 & 15,7 & 26,4 & 45,5 & 3,39 & 2,84 & 2,74 & 0 & 2,64 & 0 \\
\hline Ch103 & $457,8^{\prime}-5^{\circ} 16,7^{\prime}$ & 80 & Vase sableuse/ 5gy3-2 & 0,422 & 3,16 & 24,7 & 54,5 & 20,03 & 2,74 & 2,25 & -1 & 1,69 & $-0,42$ \\
\hline Ch104 & 458,9' - 5918,2' & 65 & Crème de vase/ 5gy3-2 & 0 & 0 & 0 & 0 & 0 & - & - & - & - & - \\
\hline Ch105 & $500,4^{\prime}-5916,7^{\prime}$ & 50 & Vase silteuse/ 5gy3-2 & 0 & 0 & 0 & 0 & 0 & - & - & - & - & - \\
\hline Ch106 & $502^{\prime}-596,85^{\prime}$ & 20 & Vase silteuse fluide/ 5gy 3-2 & 0 & 0 & 0 & 0 & 0 & - & - & - & - & - \\
\hline
\end{tabular}

* Coloration des sédiments (Munsell color system) 5g2-1: Noir verdâtre, 5gy3-2: Vert olive grisâtre, 10yr5-4: Brun jaunâtre modéré

${ }^{*}$ Proportion (en \%) granulométrique : Sable très grossier-Stg, Sable grossier-Sg, Sable moyen-Sm, Sable fin-Sf, Sable très fin-Stf

${ }^{* * *}$ Paramètres granulométrique : Md-Médiane, M-Moyenne, Sk-Asymétrie, So-Classement, Mo-Mode 


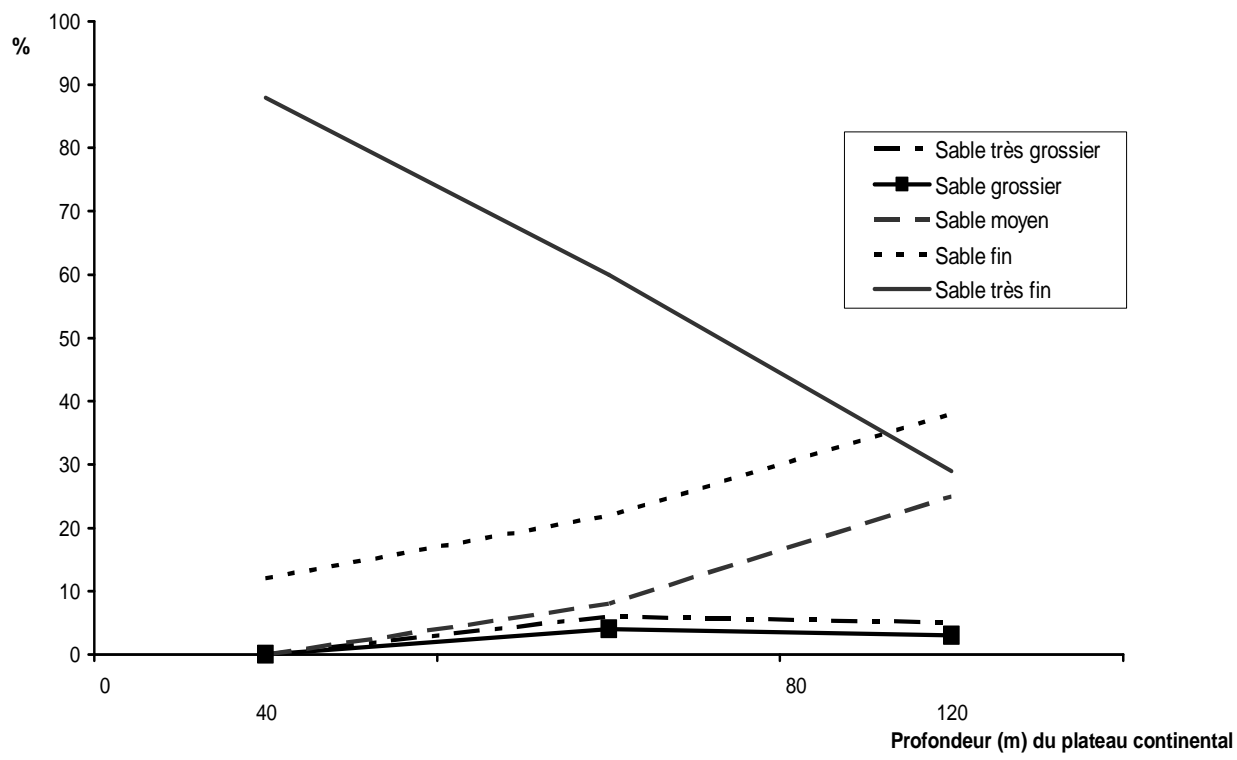

Figure 2a : Répartition des faciès sableux du plateau continental au large de Dabégo

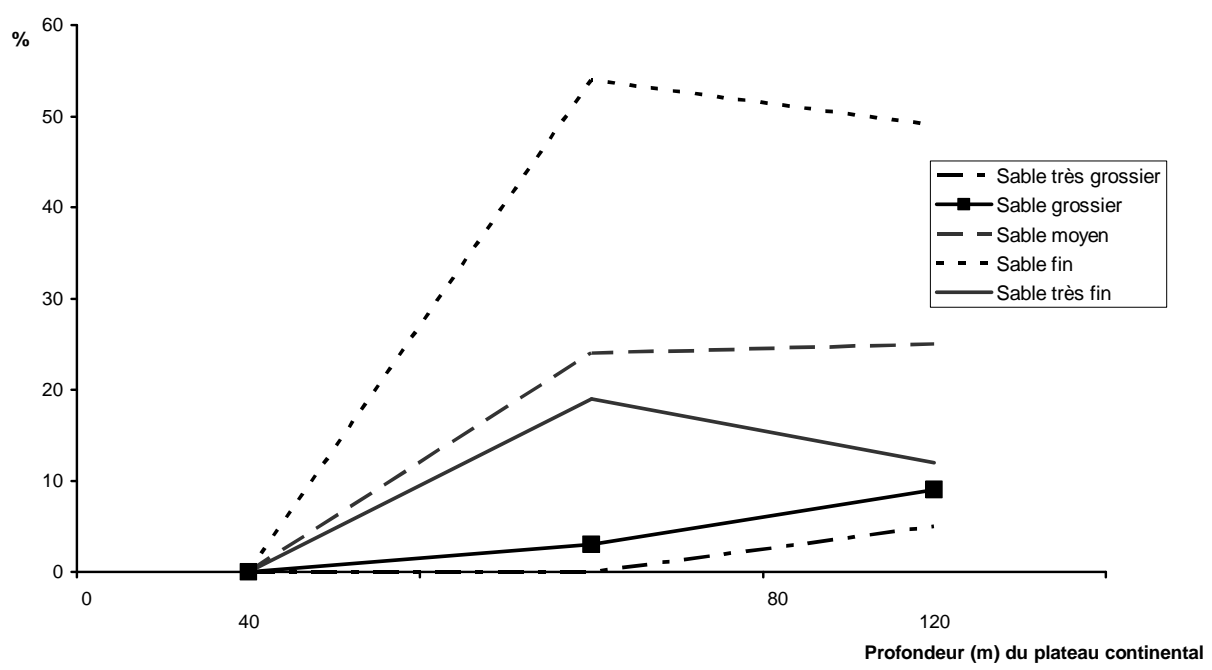

Figure $2 \mathrm{~b}$ : Répartition des faciès sableux du plateau continental $\mathrm{au}$ large d'Alékedon 


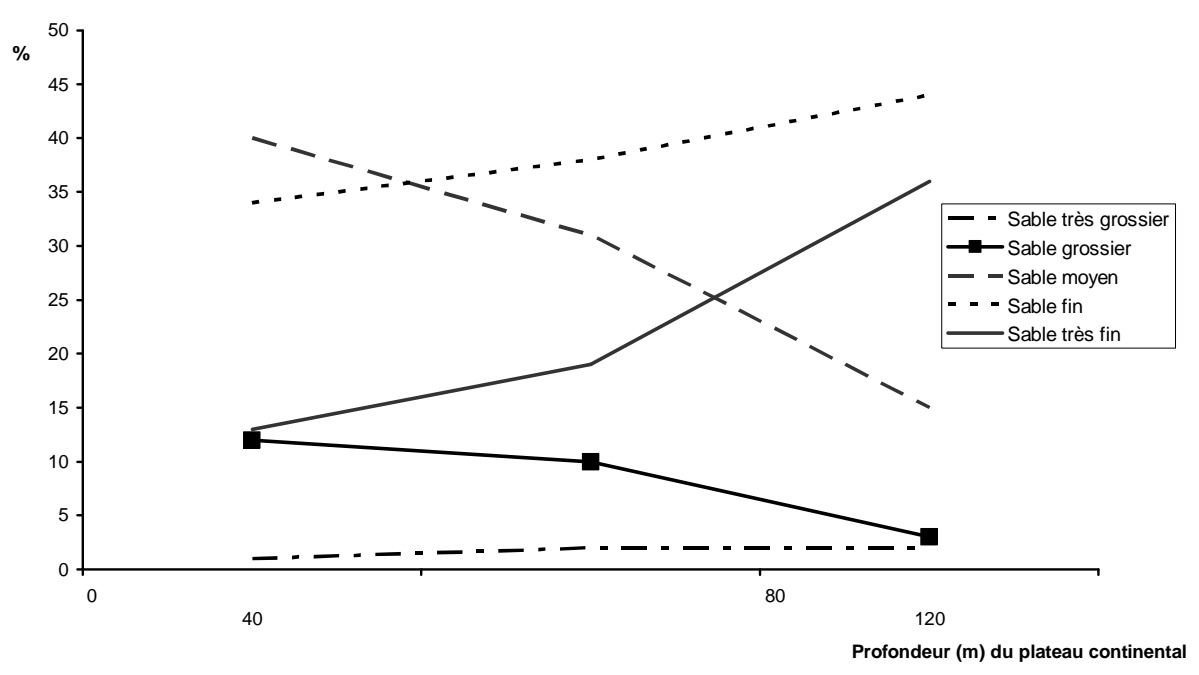

Figure 2c : Répartition des faciès sableux du plateau continental au large de Fresco

Cette distribution granulométrique, dans les radiales de Dabégo, d'Alékedon et de Fresco permet de faire deux remarques :

(i) une absence de classement des sables vaseux très fins et des vases sableuses très fines qui s'explique par le morphodynamisme des environnements sédimentaires à barres de grès [5, 13],

(ii) des apports de sédiments fins dans les ensembles morphobathymétriques concaves.

Dans ces zones de non-apports sédimentaires, les courants généraux, la marée et la houle induisent un dynamisme permettant la mise en suspension et le transport de sédiments fins au-delà du plateau continental interne où il ne reste plus que les sédiments à grains grossiers $[14,15]$.

Les paramètres de distribution granulométrique montrent que, dans les sables vaseux, les grains sont médiocrement classés, en moyenne mal triés et ont une asymétrie vers les plus petites tailles. II s'agit, en accord avec l'interprétation de Friedman [16], de sables vaseux d'origine fluviatile, accumulés sur le plateau continental. 
Ces sables sont prépondérants dans les sédiments «mixtes» et proviendraient des altérites du socle précambrien de l'arrière pays continental par le biais des cours d'eau et des cordons littoraux fossiles ou actuels et de sables quaternaires [17, 18].

Les tendances de dispersion des sables des ensembles bathymétriques concaves du plateau continental (Figure 3), montrent que les grains moyens (M) se situent entre 0 et 2,75. Le classement (So) varie entre 0,9 et $+0,1$. L'asymétrie (Sk) fluctue de $-0,3$ à $+0,2$ et la médiane $(\mathrm{Md})$, entre 0 et 2,85. Ces paramètres indiquent également que les grains moyens sont inférieurs à 3,2 (Figure 3a), le classement est inférieur à 0,8 (Figure 3b), l'asymétrie est inférieure à 0,5 (Figure 3c) et la médiane est inférieure à 3,2 (Figure $3 d$ ). Ces résultats peuvent être interprétés selon Friedman [19] et Stewart [20] comme des dépôts de ligne de rivage, c'est à dire une zone de plate-forme soumise à un paléocourant tidal.

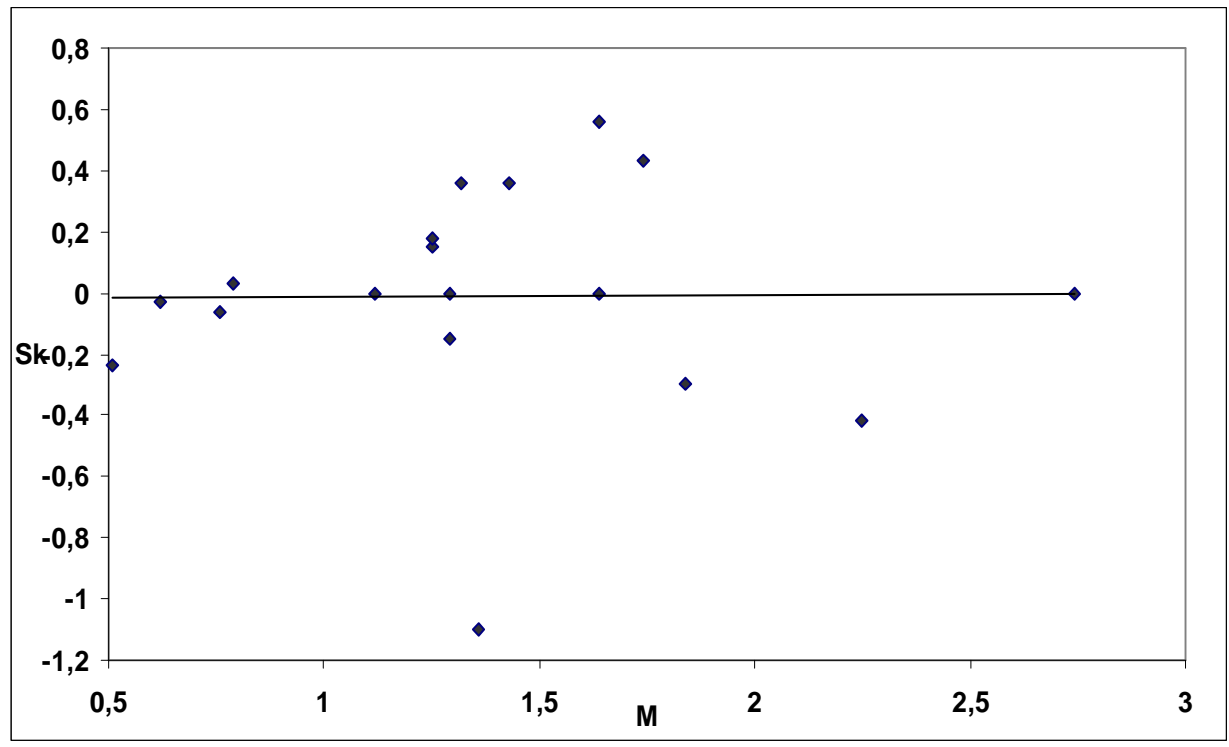

Figure 3 a: Evolution des tendances asymétrie / grain moyen des sables 


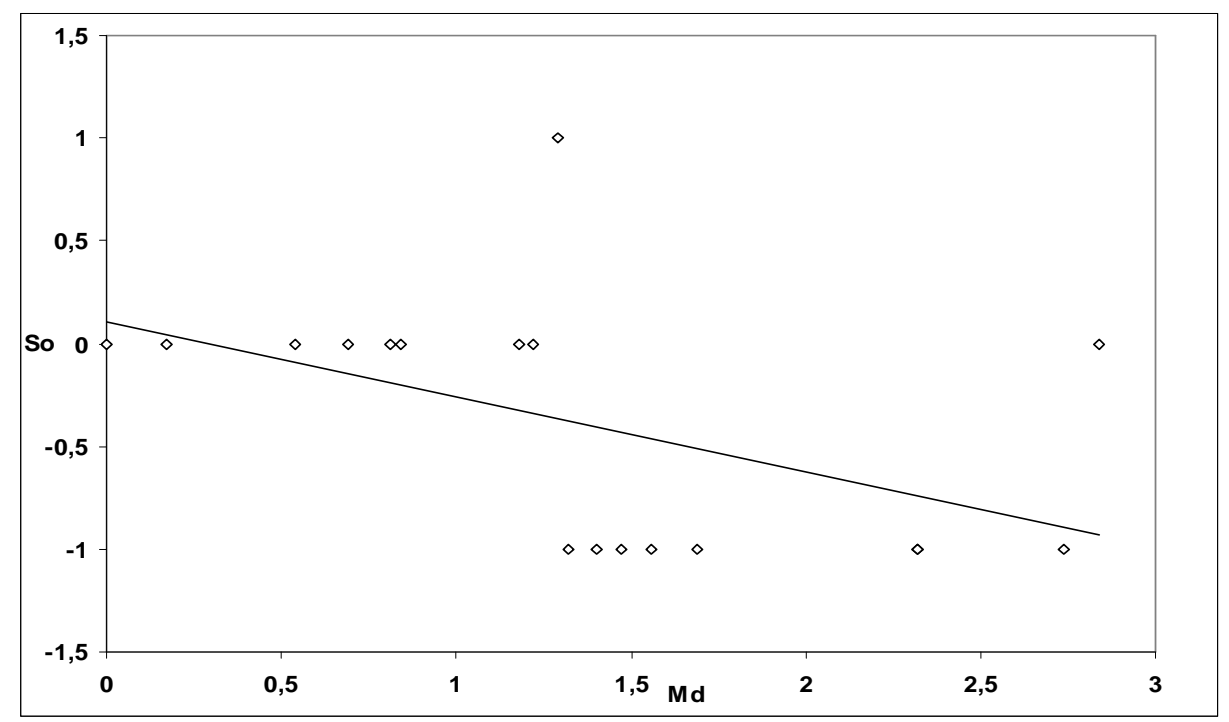

Figure $3 \mathbf{b}$ : Evolution des tendances classement / médiane des sables

\section{3-2. Analyse pétrographique des beach-rocks du plateau continental de Côte d'Ivoire}

Les échantillons étudiés sont des arénites d'aspect consolidé à texture hétérogranulaire. Les grains de sables sont de taille variable comprise entre 2 et 0,063 mm. Ces sables sont accompagnés de débris d'organismes. Les roches sont parsemées de cavités traduisant une porosité intergranulaire [10]. Elles résulteraient de l'altération superficielle conduisant au départ de certains constituants. Dans ce cas, ces cavités sont post-sédimentaires.

Les grains de quartz analysés sont automorphes, arrondies, translucides et non altérés et présentent un éclat gras. Ces grains de quartz sont pris dans un ciment ferrugineux de couleur sombre et devenant faiblement carbonaté par endroits.

L'étude des beach-rocks au microscope polarisant présente des éléments détritiques, bioclastiques pris dans un ciment à nombreux pores intergranulaires. La roche observée a une texture réticulée traduite par des grains non jointifs. Seuls les cristaux des grains polycristallins sont unis les uns aux autres donnant des contacts imbriqués. 


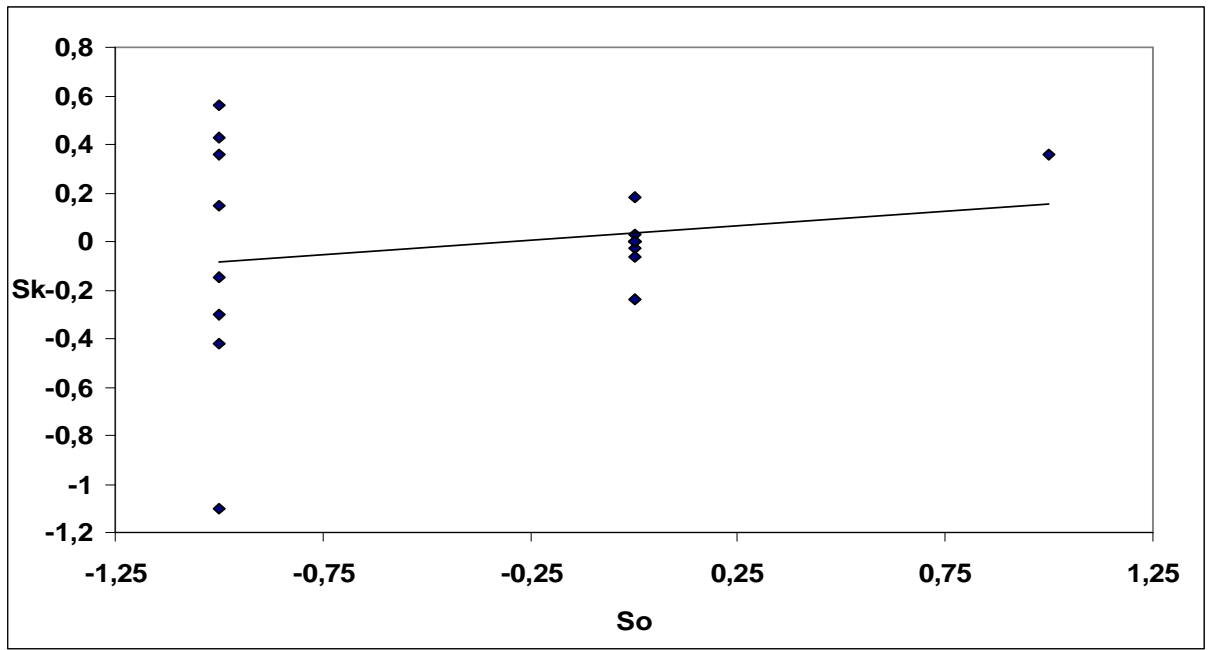

Figure 3c : Evolution des tendances asymétrie / classement des sables

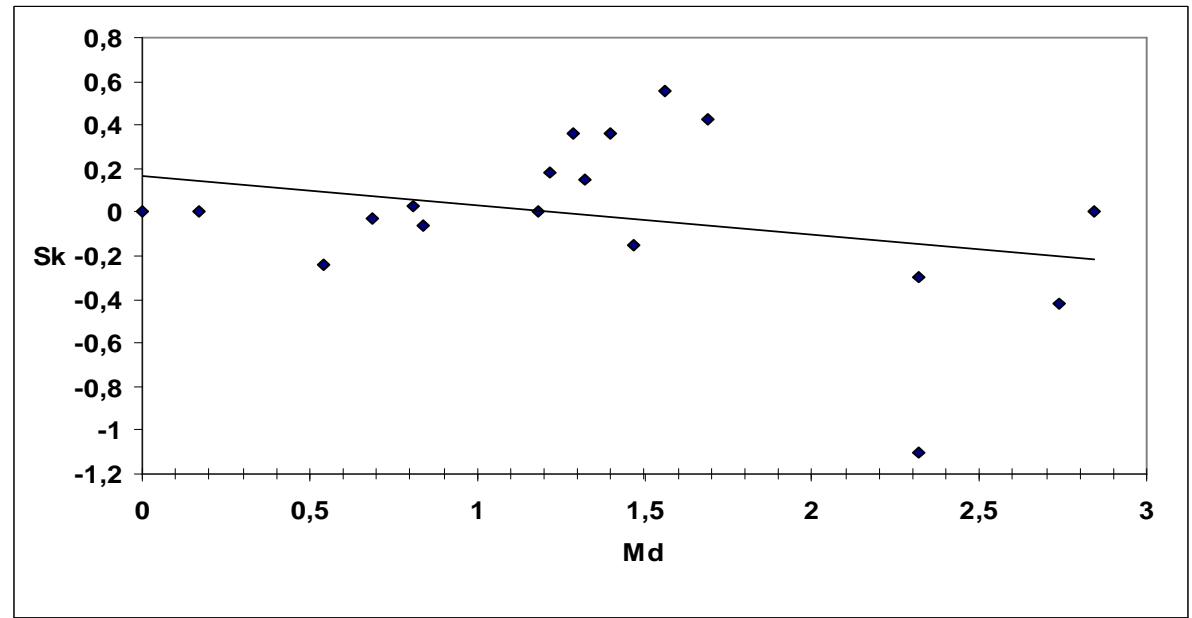

Figure 3d : Evolution des tendances asymétrie / médiane des sables

\section{3-2.1. Caractérisation des éléments figurés}

Ils sont composés d'espèces minérales et de débris d'organismes (Photo 1). Le tableau 2 présente leur description au microscopique polarisant. Les espèces minérales sont constituées par ordre d'abondance de quartz, de feldspath et de lithite.

(i) le quartz se présente sous deux aspects : le quartz monocristallin et le quartz polycristallin (Photo 2). Les grains de quartz présentent quelques 
fois des extinctions roulantes. Ce qui indique une déformation du minéral; (ii) les grains de feldspath rencontrés sont principalement de l'orthose et de l'albite. Ils sont en faible proportion par rapport au quartz, (iii) les lithites présentent de rare muscovite. La biotite est également rare et se retrouve en fragment. L'opale se présente en inclusion dans certains grains de quartz.

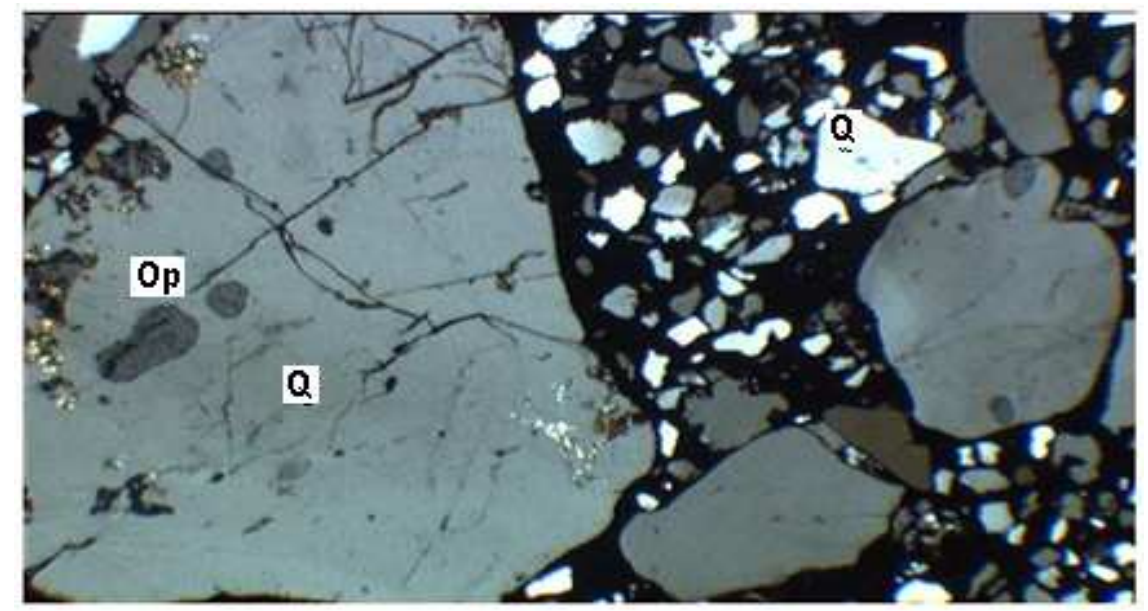

Photo 1a : Inclusion d'Opale (Op) dans des phénocristaux de Quartz (Q). Grès hétérogranulaire (grossissement $x$ 100, LP)

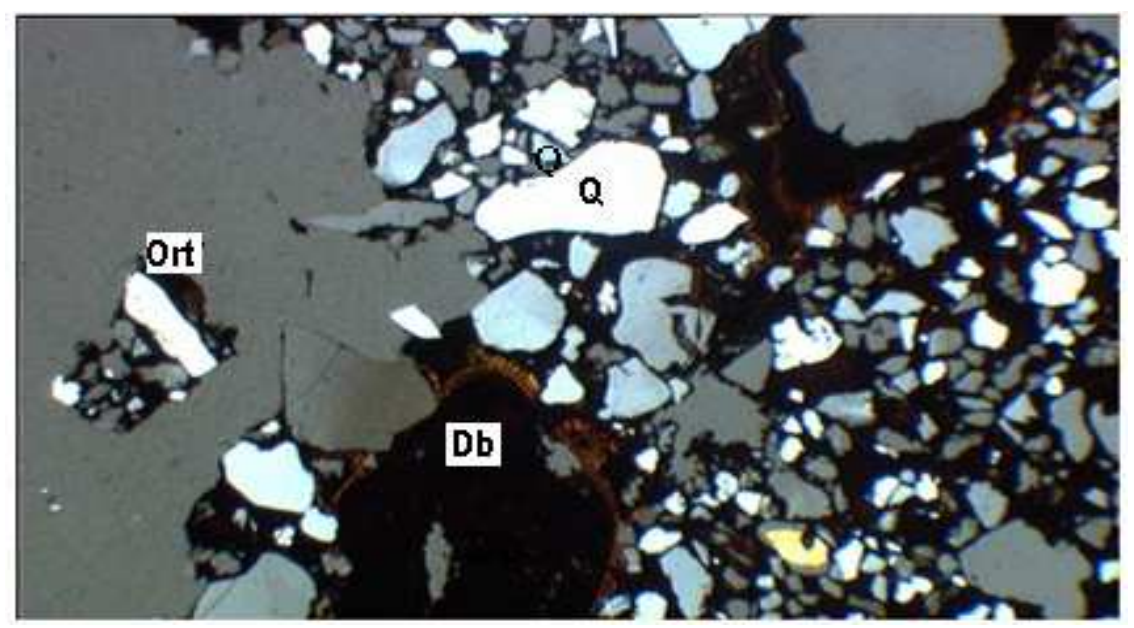

Photo 1b: Eléments bioclastiques et détritiques. Débris bioclastiques $(D b)$. Orthose (Or) à mâcle simple et Quartz (Q) dans du grès hétérogranulaire $(G \times 100, L P)$ 


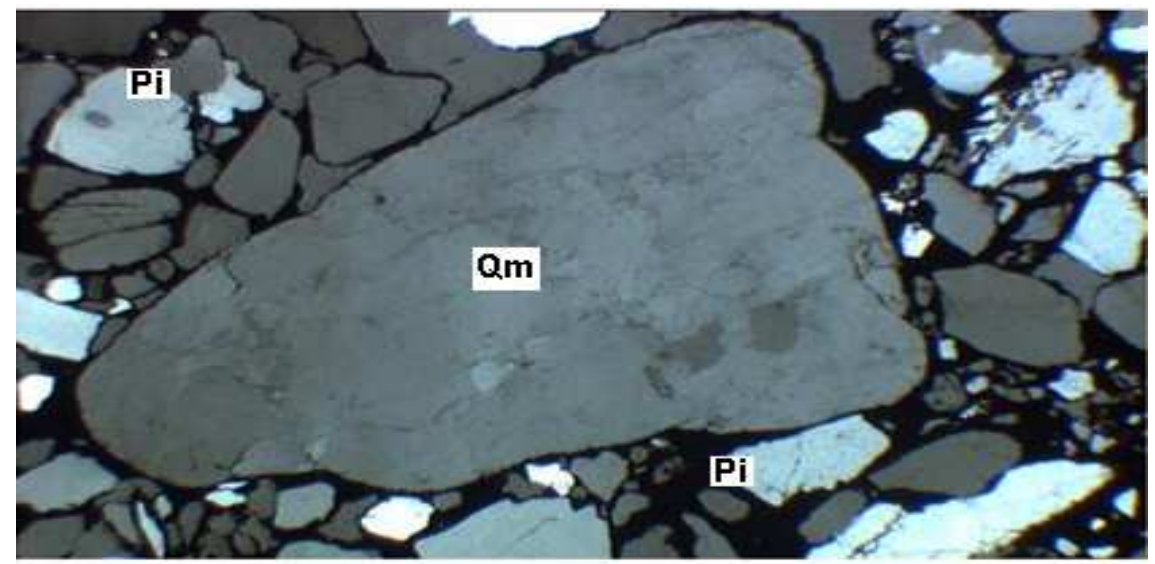

Photo 2a : Porosité intergranulaire (Pi) du grès hétérogranulaire et à phénocristaux de Quartz monocristallin (Qm) anguleux (G x 100, LP)

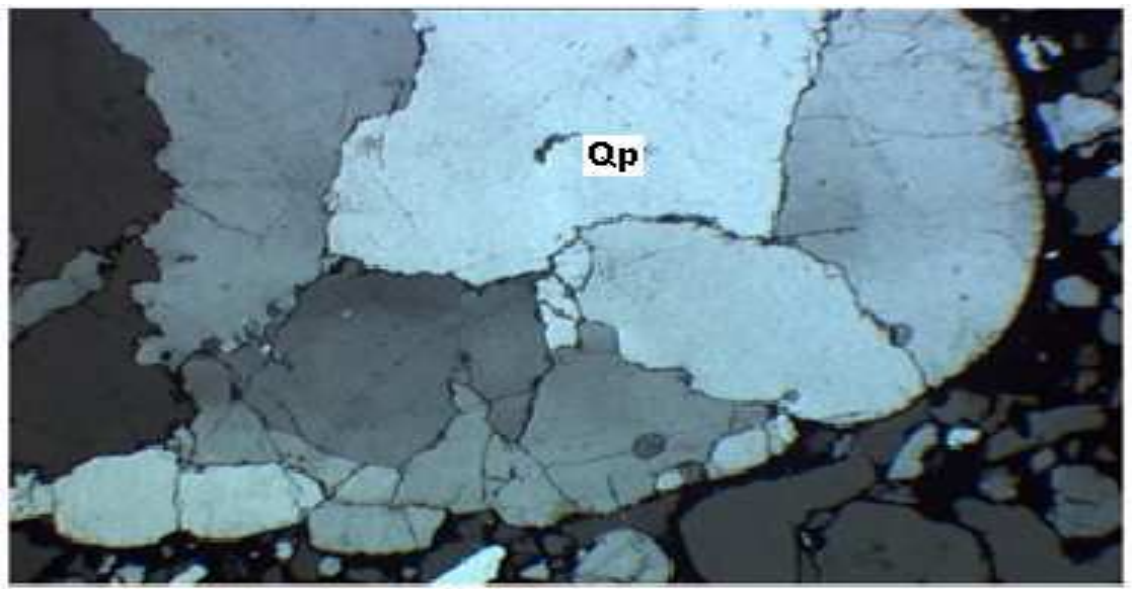

Photo 2b: Phénocristaux de Quartz polycritallin (Qp) subanguleux du grès hétérogranulaire $(G \times 100, L P)$

Les caractères sédimentologiques des éléments figurés sont définis par l'aspect, la taille, la forme et la fragmentation des grains, l'influence du transport sur les particules, la présence d'éléments à signification paléoenvironnementale et la présence de cavité. Le quartz présente des grains monocristallins et des grains polycristallins. Les grains monocristallins représentent 90 contre $10 \%$ pour les grains polycristallins. 
L'état polycristallin provient d'une recristallisation. Ils sont en général bien roulés et présentent par endroits des cassures incorporant le ciment.

Tableau 2 : Description minéralogique des beach-rocks au microscope polarisant

\begin{tabular}{|c|c|c|}
\hline Minéraux & $\begin{array}{l}\text { Observation en lumière } \\
\text { naturelle }\end{array}$ & Observation en lumière polarisée \\
\hline Quartz & $\begin{array}{l}\text { Automorphes et incolores } \\
\text { Transparents et limpides } \\
\text { Relief faible et cassures } \\
\text { Inclusions de minéraux }\end{array}$ & $\begin{array}{l}\text { Biréfringence faible } \\
\text { Polarise dans le gris-très claire } \\
\text { d'ordre I } \\
\text { Extinctions roulantes }\end{array}$ \\
\hline Orthose & $\begin{array}{l}\text { Incolore et automorphe } \\
\text { Relief faible } \\
\text { Altéré }\end{array}$ & $\begin{array}{l}\text { Biréfringence faible } \\
\text { Polarise dans le gris très clair d'ordre } \\
\text { I. } \\
\text { Macles de carlsbad. }\end{array}$ \\
\hline Albite & $\begin{array}{l}\text { Incolore et automorphe } \\
\text { Relief faible } \\
\text { Altéré }\end{array}$ & $\begin{array}{l}\text { Biréfringence est faible } \\
\text { Polarise dans le gris-très claire } \\
\text { d'ordre I } \\
\text { Macle simple de l'albite }\end{array}$ \\
\hline Muscovite & $\begin{array}{l}\text { Incolore et limpide } \\
\text { Automorphe (petites paillettes) } \\
\text { Relief moyen et bon clivage }\end{array}$ & $\begin{array}{l}\text { Biréfringence forte } \\
\text { Teinte vive de polarisation d'ordre II } \\
\text { Extinction droite }\end{array}$ \\
\hline Biotite & $\begin{array}{l}\text { Automorphe } \\
\text { Transparent coloré (vert } \\
\text { jaunâtre) } \\
\text { Relief moyen. }\end{array}$ & $\begin{array}{l}\text { Biréfringence forte } \\
\text { Teinte vive de polarisation d'ordre II. }\end{array}$ \\
\hline Opale & $\begin{array}{l}\text { Incolore et forme circulaire } \\
\text { Inclusion dans du quartz }\end{array}$ & Éteint et isotrope. \\
\hline
\end{tabular}

Les éléments figurés des barres de grès se regroupent en quatre classes granulométriques (Tableau 3). II s'agit de la classe des fins, des moyens, des grossiers et des très grossiers. Les sédiments sont généralement sableux de taille variable. Les éléments très grossiers sont en faible proportion (10\%). Les éléments moyens sont aussi arrondis et 
subanguleux. Par contre, les grains fins sont en général anguleux parfois subanguleux.

Tableau 3 : Granulométrie et morphoscopie des éléments figurés

\begin{tabular}{lll}
\hline Classes granulométriques & Proportion (\%) & Morphoscopie \\
\hline Fins & 30 & Subanguleux \\
Moyens & 35 & Arrondis \\
Grossiers & 25 & Anguleux \\
Très grossiers & 10 & Anguleux \\
\hline
\end{tabular}

\section{3-2-2. Ciments des beach-rocks}

Le ciment des barres de grès consiste en une précipitation d'oxyde de fer, de couleur brune en LN. Cependant en LP, il est sombre et isotrope. On observe par endroits dans le ciment l'aragonite de couleur brune, présentant en LP, une teinte rouge avec des irisations. Ce minéral s'observe quelques fois tout autour des cavités et participe par endroits à la substitution du ciment ferrugineux par un ciment carbonaté s'observe avec une incrustation du ciment dans les débris organiques (Photo 3). Cette substitution s'expliquerait par la diagenèse avancée traduisant la maturité des barres de grès [21].

L'analyse sédimentologique montre que le ciment est ferrugineux et non cristallisé. II est issu de la précipitation des minéraux d'oxyde de fer authigènes. Un ciment s'observe entre les cassures des grains de quartz. L'incorporation du ciment montre la postériorité de sa formation par rapport au dépôt sédimentaire. II occupe une proportion de $20 \%$ par rapport à l'ensemble de la roche. Le ciment ferrugineux est substitué par endroits par l'aragonite. 


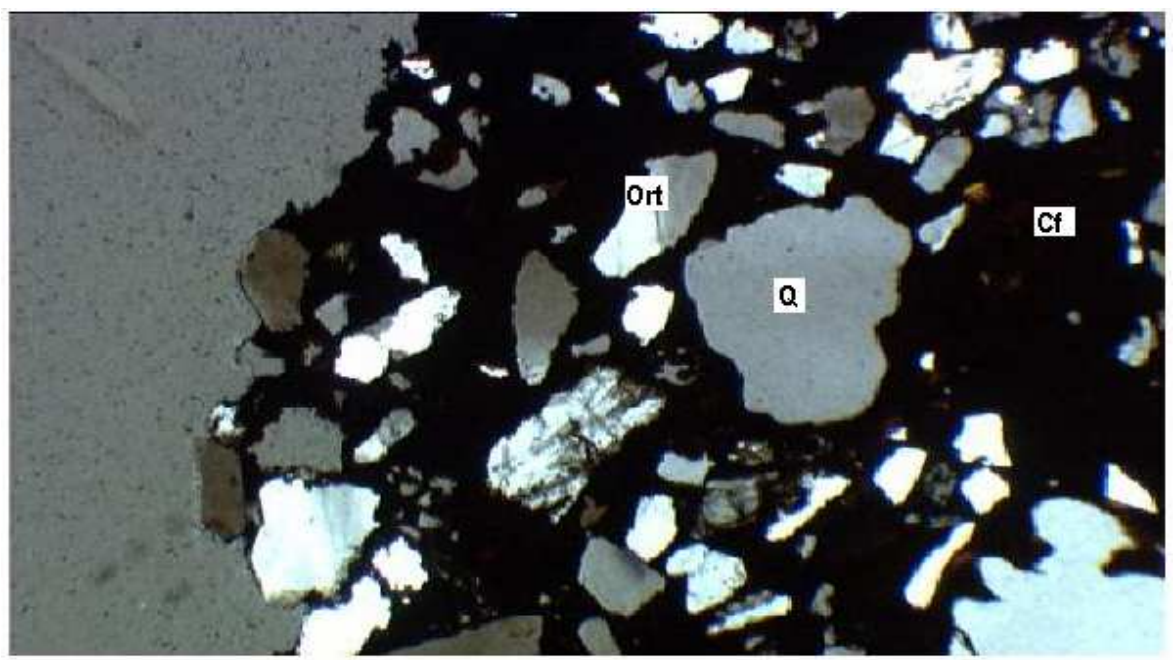

Photo 3: Texture hétérogranulaire d'un grès. Orthose (Ort) à mâcle simple, phénocristaux de Quartz (Q) et ciment ferrugineux (Cf)- $(G x$ 100, LP)

\section{3-2-3. Classification, origine et mode d'accumulation des barres de grès}

Dans le diagramme de Dott-Pettijohn [11, 12], les proportions relatives des minéraux des beach-rocks sont en moyenne de $90 \%$ pour le quartz, $5 \%$ pour le feldspath et $5 \%$ pour les lithites (Figure 4). Ces proportions placent ces beach-rocks dans le domaine des quartzarénites ou grès quartzeux. Par ailleurs, selon la classification de Cailleux [10] alliant la nature minéralogique et le ciment, les beach-rocks étudiés sont des grès quartzeux à ciment ferrugineux.

Les grains de quartz analysés présentent des extinctions roulantes, traduisant une déformation tectonique. Ces quartzs à extinction roulante sont issus de la désagrégation de roches métamorphiques et non d'origine magmatique (quartz dépourvus d'extinction onduleuse). Les sables consolidés sont d'origine terrigène et, résultent de l'érosion des altérites issues du socle. Le ciment ferrugineux proviendrait de la recristallisation secondaire de l'oxyde de fer recouvrant les sables grossiers formant le cordon littoral [22, 23]. 
Les grains, dans les lames minces, sont dispersés. Cette disposition, sans direction préférentielle des grains, est fréquemment le résultat d'une rapide accumulation en masse [24].

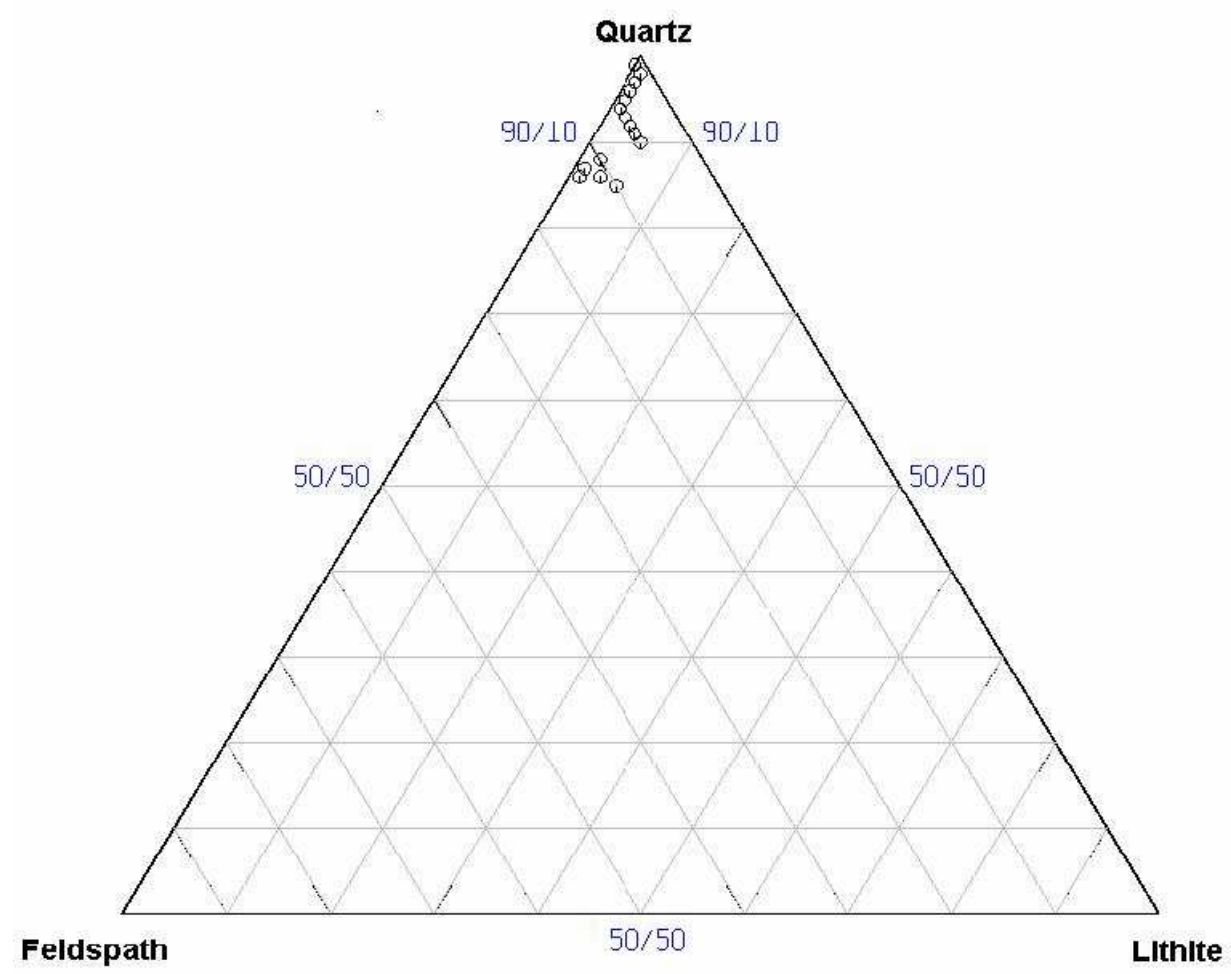

Figure 4 : Les quartzarénites du plateau continental de Côte d'Ivoire

\section{3-2.4. Evolution diagénétique des beach-rocks}

Les grains de sables sont généralement arrondis avec un éclat vif et luisant. Ces caractéristiques suggèrent des éléments transportés par les cours d'eau qui se jettent dans le plateau continental [23, 25]. Le transport pourrait également avoir été effectué très lentement dans une masse vaseuse dense [9].

En plus de leur forme arrondie, les particules ont subi une ségrégation minérale. II y a une prédominance de quartz par rapport au feldspath avec une absence d'éléments argileux. Pour Cailleux [10], l'abondance du quartz est due à la sélection minéralogique des éléments lors du 
transport. En effet, durant le transport, les quartz résistent aux actions mécaniques. Alors que les minéraux altérables comme les feldspaths, sont réduits en éléments très fins. Ces éléments argileux sont progressivement éliminés au cours du transport. Ces caractères montrent que les sédiments ont subi un long transport. Les grains de quartz fins et anguleux résulteraient de l'action mécanique exercée sur les sédiments au cours de leur déplacement. En effet, pendant le transport, les éléments s'entrechoquent et se fragmentent pour donner des grains de petite taille. Leur forme anguleuse indique que ceux-ci n'ont pas subi une longue période d'usure avant le dépôt.

Après le dépôt, les sédiments subissent un phénomène diagénétique qui peut évoluer vers le stade extrême. C'est la diagénèse avancée. Les grains polycristallins sont bien roulés car ils ont subi un long transport. Si ces grains étaient initialement polycristallins avant leur dépôt, ils se fragmenteraient par l'action mécanique du transport or ces polycristaux sont restés soudés. Ces grains polycristallins résulteraient donc de la transformation des grains monocristallins de quartz sous l'influence du phénomène de recristallisation. Les travaux de Cailleux [10] et de Saaidi [26] ont montré que certains minéraux de la roche sont dissous par les solutions interstitielles par le biais des fissures. La matière ainsi dissoute va contribuer à développer et nourrir d'autres cristaux devenant de plus en plus gros. Au terme de l'évolution, la structure primitive du dépôt est complètement modifiée. Ainsi, c'est la recristallisation des grains monocristallins qui a donné naissance aux grains polycristallins, traduisant la maturité des barres de grès étudiées.

\section{3-3. Morphodynamisme des beach-rocks}

Les sables vaseux très fins sont de type granulométrique parabolique [13], ce qui traduit leur arrêt brutal [27] lors du transport de ces sédiments au contact de ces véritables barrières ( $20 \mathrm{~m}$ de hauteur) que constituent les barres de grès au large de la Côte d'Ivoire [3, 4].

Dans le littoral du Togo, les beach-rocks ont en moyenne $2 \mathrm{~m}$ d'épaisseur et $25 \mathrm{~m}$ de largeur [2]. Les essais sur modèles réduits 
montrent qu'à plus de $2 \mathrm{~m}$ de hauteur, les beach-rocks arrêtent la quasitotalité des échanges sédimentaires et au fur et à mesure de l'abaissement de cette altitude, la restriction des transferts diminue. En outre, sa résistance mécanique varie de 250 à $350 \mathrm{~kg} \mathrm{~cm}^{-2}$ [28]. Ce sont donc des résistances élevées, souvent supérieures à celles du béton. Ces caractères suffisent pour que les beach-rocks soient intégrés dans la protection naturelle de la côte face à l'érosion du littoral.

Au Bénin, seule la zone comprise entre le Sud-Est et la frontière du Nigeria bénéficierait de l'effet de protection des beach-rocks et ralentirait l'érosion côtière [1]. L'interprétation dynamique de cette évolution serait que la barre de grès, en dispersant sur sa surface l'énergie de déferlement, ralentit et peut même bloquer les transferts sédimentaires [29].

\section{3-4. Paléoenvironnement des beach-rocks}

Les beach-rocks du Golfe de Guinée sont comparables aux " arréfices » du plateau continental brésilien décrit par Ottmann [30] quant à la nature sableuse à prédominance quartzeuse et à la présence de débris d'organismes. En outre, les beach-rocks contiennent des feldspaths, de la biotite et de la muscovite. La cimentation des arréfices s'est réalisée par la recristallisation de la calcite. La présente étude montre cependant un ciment ferrugineux substitué par endroits par un ciment carbonaté. De plus, les sables grossiers du cordon littoral actuel sont recouverts d'oxyde de fer [23]. Cela expliquerait la nature ferrugineuse du ciment formé par précipitation de l'oxyde de fer recouvrant les sables grossiers du littoral actuel.

La formation des beach-rocks serait liée aux phénomènes hydrodynamiques. En effet, la cimentation s'effectue dans les environnements de dépôt non turbulents. Les zones agitées sont dépourvues de beach-rocks [2, 13]. Les barres de grès se sont formées dans un environnement marin. La majorité des éléments est arrondie et possède une surface propre avec un éclat vif. Ils ont subi un long transport. Ces sables sont triturés en mer par les marées et les vagues 
[10]. Ces dépôts sableux se sont consolidés pour donner les beach-rocks [13].

Dans le cas du Togo, des datations de coquilles permettent de préciser l'évolution de la dynamique dans la partie externe du cordon. Elles attribuent un âge de $3800 \pm 90$ B.P aux beach-rocks dont l'altitude est comprise entre 1 et $1,5 \mathrm{~m}$ et de $2890 \pm 100$ B.P au matériel du cordon qui le surmonte à $+3 \mathrm{~m}$. On peut donc penser que les beach-rocks se sont formés lors du recul d'un trait de côte lié à une transgression, ou à une stabilisation, et qu'il a été ensuite fossilisé par les crêtes de plage successives qui se sont déposées lors de la régression qui a suivi. Les transgressions ont dû être de faible amplitude puisque les dépôts actuels atteignent $+4,5 \mathrm{~m}$ et que l'altitude maximale du cordon ancien du Togo est de $+6,5 \mathrm{~m}[29,31]$.

En accord avec Ottmann [30]; Martin [3], Tastet [5] et Aka [6], ce travail montre que les barres de grès sont des cordons littoraux fossiles résultant d'une évolution de l'environnement sédimentaire sous la dépendance des variations du niveau de la mer et des fluctuations climatiques. Leur environnement de dépôt est donc une plage.

\section{Conclusion}

Les beach-rocks du golfe de Guinée sont essentiellement des quartzarénites ou des grès quartzeux à ciment ferrugineux et carbonaté par endroits. De plus, les éléments figurés sont accompagnés de débris d'organismes remplis par le ciment. C'est le phénomène d'incrustation. II subsiste toutefois des cavités qui traduisent leur degré de perméabilité. Ces barres de grès sont formées par consolidation de matériaux terrigènes déposés à l'issue d'un long transport fluvial, traduisant ainsi leur maturité. La fraction grossière de la couverture sédimentaire dans les zones à morphologie concave montre une absence de classement granulométrique, liée aux barres de grès. Le faciès granulométrique parabolique a été reconnu sur le plateau continental de Côte d'Ivoire car les barres de grès constituent de véritables obstacles au transport des sédiments. Les beach-rocks ralentissent la quasi-totalité des transferts 
sédimentaires et par conséquent, stabilise le trait de côte sur les littoraux du Togo et du Bénin. Son intérêt réside donc dans le fait qu'il assure la protection naturelle des plages contre l'érosion côtière dans le Golfe de Guinée.

\section{Remerciements}

Nous exprimons nos remerciements au Département des Sciences de la Terre de l'Université de la Rochelle (France) et au Centre de Recherches Océanologiques d'Abidjan

\section{Références}

[1] - L.GNONLONFIN, H. GNONLONFOUN et K.S. ADAM. Etat et suivi du littoral béninois. In: Actes du colloque érosion côtière Lomé, 19-22 septembre 1998, (1988) pp.2-28

[2] - A. BLIVI. Un élément de la dynamique littoral : Le beach-rock. In : Actes du colloque régional érosion côtière, Lomé, 19-22 septembre 1988, (1988) pp.135-151.

[3] - L. MARTIN. Morphologie, sédimentologie et paléogéographie au Quaternaire récent du plateau continental ivoirien. Thèse Doctorat Etat, Université de Paris VI, (1973) 340p.

[4] - S. MONDE. Nouvelles approches de la cartographie du plateau continental de Côte d'lvoire: Aspects morphologiques et sédimentologiques. Thèse Doctorat 3ème cycle, Université d'Abidjan, (1997) 175p.

[5] - J.P. TASTET. Environnements sédimentaires et structuraux quaternaires du littoral du golfe de Guinée (Côte d'Ivoire, Togo et Bénin). Thèse Doctorat Etat, Université de Bordeaux I (1979). 175p.

[6] - K. AKA. La sédimentation quaternaire sur la marge de Côte d'Ivoire : Essai de modélisation. Thèse Doctorat Etat, Université de Côte d'Ivoire, (1991) 320p. 
[7] - S. MONDE, K. AKA, G. JOSEPH et O. LEENHARDT. Nouvelles cartes bathymétriques et sédimentologiques du plateau continental de Côte d'Ivoire. Géologues 117 (1998) 17-23.

[8] - J. MACMANUS. Grain size determination and interpretation In: Techniques in Sedimentology, Tucker Edition, Blackwell Scientific Publ., 0xford, (1988) pp.63-85

[9] - H. CHAMLEY. Properties of sedimentary particles In : Sedimentology. Edition Chamley, Springer-Verlag, Berlin, (1990) pp.44-59.

[10] - A. CAILLEUX. Distinction des sables marins et fluviatiles. Bull. Soc. Géol. Fr., 5 XV : (1947) 375-404.

[11] - R.H. DOTT. Wacke, greywacke and matrix - what approach to immature standstone classifcation. J. Sedim. Petrol. 34 : (1964) 625-635.

[12] - F.J. PETTIJOHN, P.E. POTTER \& R. SIEVER. Sand and sandstone. Spinger Edition, New York, Heidelberg, Berlin (1972), 618p.

[13] - S. MONDE et K. AKA. Analyse sédimentologique et paléoenvironnements des sédiments superficiels des ensembles morphobathymétriques du plateau continental de Côte d'lvoire. Afr. Geosci. Rev. 8 , 4 : (2001) 413-423.

[14] - N. PLUTCHART. Guinea current In: The Encyclopaedia Oceanographic. Fairbridge Edition, New York, Reinhold, (1966) pp.311-312.

[15] - L. LEMASSON et J.P. REBERT. Circulation dans le golfe de Guinée. Etude de la région d'origine du sous-courant ivoirien. Cah. Orstom, Sér. Océan. II : (1973) 303-316.

[16] - G.M. FRIEDMAN. Distinction between dune, beach and river sands from their textural characteristics. J. Sedim. Petrol. 31 : (1961) 514-529.

[17] - N. LENEUF. Le continental terminal. Contribution à l'établissement de la notice explicative de la carte géologique au 1/1.000.000 de la Côte d'Ivoire. Université d'Abidjan-Sodemi, Rapp. Sodemi 204, (1968) 37p.

[18] - B. LEBUANEC. Les principaux sols de Côte d'lvoire et leurs aptitudes culturales. Ann. Univ. Abidjan 8, 2 : (1972) 17-26.

[19] - G.M. FRIEDMAN. By name process and statistical parameters compared for size frequency distribution of beach and river sands. $J$. Sedim. Petrol. 32 : (1967) 15-25. 
[20] - H.B.J. STEWART. Sedimentary reflections on depositional environments in San Migue Lagoon, Baja, California, Mexico. Bull. Am. Assoc. Petrol. Geol. 42 (1958) : 2567-2618.

[21] - R.L. FOLK. Stages of textural maturity in sedimentary rocks. J. Sedim. Petrol. 21 : (1951) 127-130.

[22] - B. TAGINI. Esquisse structurale de la Côte d'Ivoire. Essai de géotectonique régionale. Thèse Doctorat Etat, Université de Lausanne (1971), 302p.

[23] - L. MARTIN. Morphologie, sédimentologie, paléogéographie au Quaternaire du plateau continental ivoirien. Trav. Doc. Orstom 61, (1977) 65p.

[24] - PA. SCHOLLE \& D. SPEARING. Sandstone depositional environments. Bull. Am. Assoc. Petrol. Geol. 31 (1982) : 352-401.

[25] - J.M AVENARD, M. ELDIN, G. GIRARD et SIRCOULON. Aspect de la géomorphologie. In : Le milieu naturel de Côte d'Ivoire, Mém. Orstom 50 : (1971) 1-72.

[26] - E. SAAIDI. Roches et ensembles détritiques In : Traité de sédimentologie : Pétrographie des environnements sédimentaires, Edition Afrique orient (1991), pp.53-175

[27] - A. RIVIERE. Expression analytique générale de la granulométrie des sédiments meubles. Notions de faciès granulométrique. Bull. Soc. Géol. Fr. 6 (II) (1952) : 155-167.

[28] - LCHF. Influence du beach-rock sur la stabilisation du profil de plage. Essais en laboratoire. Rapp. Minist. Equip. Transp. Bénin. Proj. Erosion côtière, (1988) 11p.

[29] - G. ROSSI. Synthèse sur l'érosion du littoral dans le golfe de Bénin. In: Actes du colloque érosion côtière Lomé, 19-22 septembre 1998 (1988) pp.153-189

[30] - F. OTTMANN. Une hypothèse sur l'origine des «arrecifes» du Nordeste brésilien. C. R. Somm. Soc. Géol. Fr. 7 (1960) :175-176.

[31] - J. LANG et G. PARADIS. Le quaternaire margino-littoral béninois (Afrique de l'Ouest). Synthèse des datations au carbon 14. Paleoecol. Afr. 16 : (1984) 65-76. 